\title{
Familiarity, memorability, and the effect of typicality on the recognition of faces
}

\author{
JOHN R. VOKEY and J. DON READ \\ University of Lethbridge, Lethbridge, Alberta, Canada
}

\begin{abstract}
Typical faces are more poorly discriminated on tests of recognition than are atypical faces, an effect suggested to mediate similar findings for attractive or likable faces. We tested the hypothesis that the effect of typicality on recognition is a function of context-free familiarity and memorability, which function in opposition. Two orthogonal principal components were extracted from subjects' ratings of faces for typicality, familiarity, attractiveness, likability, and memorability-one consisting of the ratings of familiarity, attractiveness, and likability, and reflecting context-free familiarity, and the other consisting of the memorability rating. As expected, typicality loaded equally $(r \approx .66)$, but with opposite sign, on both components. In subsequent experiments, both components were found to be significant and additive predictors of face recognition with no residual effect of typicality. General familiarity decreased discrimination, and the memorability component enhanced it, supporting the hypothesis. The results are discussed in terms of the mirror effect.
\end{abstract}

The effects of structure are ubiquitous, permeating virtually every task that we perform with natural materials. Often, these effects are manifested as effects of typicality. Such is certainly the case with people's recognition of faces. Numerous studies have demonstrated that subjects discriminate atypical or unusual faces better than typical faces on tests of item recognition (e.g., Bartlett, Hurry, \& Thorley, 1984; Cohen \& Carr, 1975; Courtois \& Mueller, 1981; Going \& Read, 1974; Light, KayraStuart, \& Hollander, 1979). ${ }^{1}$ The effect occurs both as a lower rate of false-positive responses to atypical faces, and, less reliably, as an increase in hits on typical faces (see the meta-analysis of face recognition studies for this variable in Shapiro \& Penrod, 1986). This effect of typicality has been offered as an explanation for the counterintuitive findings that attractive or likable faces are recognized less well than their less attractive or less likable counterparts; faces judged to be attractive or likable are also more likely to be thought typical (Light, Hollander, \& Kayra-Stuart, 1981; Mueller, Heesacker, \& Ross, 1984). Typicality also has been suggested to mediate the rela-

\footnotetext{
This work was supported by operating grants to each of the authors from the Natural Science and Engineering Research Council of Canada, and by an initiatory grant from the University of Lethbridge Research Fund. We thank Lorna Moore for her invaluable assistance in collecting the data, and Reid Felske of Josten's National School Service for providing copies of the high school yearbooks from which the photographs of the faces were obtained. We also thank James Bartlett, Murray Glanzer, Vicki Bruce, Margaret Jean Intons-Peterson, and two anonymous reviewers for their thorough and useful comments on an earlier version of this article. Portions of this work were presented at the annual convention of the Canadian Psychological Association, 1990. Correspondence should be addressed to J. $\mathbf{R}$. Vokey, Department of Psychology, University of Lethbridge, Lethbridge, AB, Canada T1K 3M4 (e-mail: vokey@hg.uleth.ca).
}

tionship between sex of face and face recognition. Female faces have generally been found to be recognized less well than male faces, and also to be rated as more typical than male faces (Vokey \& Read, 1988). Typicality has been found to affect the time that subjects require to identify familiar faces as known; typical faces take longer to identify (Valentine \& Bruce, 1986a). For initially unfamiliar faces, this difference in reaction time to typical and atypical faces has been found to remain large and unchanged even after 15 or more successive exposure trials (Ellis, Shepherd, Gibling, \& Shepherd, 1988).

Exactly how the categorical structure of faces produces these effects is still a matter of some debate (see, e.g., Bartlett et al., 1984; Light et al., 1979; Valentine \& Bruce, $1986 \mathrm{a}, 1986 \mathrm{~b}$; Vokey \& Read, 1988). One possibility is that the effects of category structure are mediated by distinctiveness. Thus, for example, atypical faces are more memorable because they are more distinctive than typical faces (e.g., Going \& Read, 1974); although the precise mechanism is still unclear, the greater distinctiveness of atypical faces presumably leads to greater distinctiveness in the encoding of the faces and, thereby, to enhanced retrieval (Winograd, 1981). It is true that subjects report atypical faces to be more distinctive than typical faces. Light et al. (1979) asked subjects to rate the interitem similarity of a set of faces and found that rated typicality and interitem similarity were highly correlated. Furthermore, interitem similarity proved to be a better predictor of recognition discrimination than did typicality. Moreover, Light et al. (1979) found that atypical target faces gained more for subsequent recognition from elaborative or more lengthy encoding tasks than did typical faces, presumably because these tasks increased the utilization of the distinctiveness of the atypical faces. Despite these successes, encoded distinctiveness does not appear to be the complete 
story, particularly as it fails to explain the markedly lower rate of false-positive responses to atypical faces (see, e.g., Bartlett et al., 1984; Light et al., 1979).

An alternative to an account based on distinctiveness is the idea that differences in typicality are experienced as differences in context-free familiarity that for recognition fail to be discriminated from the differences in episodic familiarity derived or derivable from specific experience with a face (see, e.g., Bartlett et al., 1984; Vokey \& Read, 1988). That is, the feelings and effects of familiarity do not directly index source (see, e.g., Mandler, 1980). Thus, the lower rate of false-positive responses to atypical faces, for example, is a direct function of the correspondingly lower level of structurally induced familiarity that these faces would have (cf. Light et al., 1979; Valentine \& Bruce, 1986b). There is some support for this hypothesis. Unfamiliar atypical faces are judged to be less generally familiar than are unfamiliar typical faces when a generally familiar face is defined to subjects as one that they may have seen before, such as on a city street (Bartlett et al., 1984), or as one that might be confusable with that of someone they know (Vokey $\&$ Read, 1988). Furthermore, familiarizing the faces has been found to ameliorate or to eliminate entirely the effect of typicality on recognition false positives (Bartlett et al., 1984). To account for the enhanced recognition discrimination of atypical over typical faces according to this notion, Bartlett et al. proposed that the increment in familiarity arising from specific prior exposure is negatively related to a face's level of structurally induced familiarity. In support of this idea, they found that, indeed, typical faces do appear to gain less for recognition from specific familiarity than do atypical faces.

To explain the effect of typicality strictly in terms of familiarity does not appear to give the whole story, either. Vokey and Read (1988), for example, reported that although typical and atypical faces do differ a priori in familiarity, these differences in familiarity may not be mediating the effect of typicality on subjects' recognition judgments. They found that although ratings of general familiarity and recognition false positives were both related to the rated typicality of the faces, they were not related to each other, although this result may have been a function of how general familiarity was defined to the subjects. Valentine and Bruce (1986a) found that typicality effects are evident even with highly familiar faces, and Ellis et al. (1988) found for initially unfamiliar faces that these effects persist over multiple exposures. Both results by themselves suggest that an explanation in terms of simple familiarity is insufficient. Valentine and Bruce (1986a) found further that the effects of typicality (rated distinctiveness in this case) and of rank order familiarity of the faces on the time to identify the faces as known were independent; both variables were significant predictors of reaction time but were unrelated to each other.

Such results do much to commend an approach to typicality effects that incorporates both distinctiveness and general familiarity. Light et al. (1979) suggested just such a model. They argued from the results of their series of experiments that at least two components were needed: a component of general familiarity derived from the structure of the similarity space of the faces and responsible for the effect of typicality on false alarms, and a specificmemory component that arises from differences in the distinctiveness of encoding of typical and atypical faces and that is responsible for the effect of typicality on hits. We also will argue for a two-component model of typicality effects on the recognition of faces. Like Light et al. (1979), we will suggest that one of the components is general familiarity. We will argue, however, that the second component is characterized better as memorability, of which the retrieval aspects of encoding distinctiveness emphasized by Light et al. for target faces are only a part. We will present data to suggest that subjects' recognition responses also reflect a judgment about the likelihood that a given distractor face would give rise to a retrieval experience if it had in fact been encountered previously. That is, the memorability of a face also has consequences for the reduction in false alarms to atypical faces. As we see it, general familiarity and memorability usually work in opposition in determining recognition, with general familiarity serving to reduce discrimination, while memorability enhances it. First, however, we present the results of an experiment in which typicality is decomposed into two independent components.

\section{EXPERIMENT 1 \\ Typicality as Familiarity and Memorability}

In a previous experiment (Vokey \& Read, 1988), we used the correlations between subjects' concurrent judgments about various attributes of faces as a method of determining the source of the effect of typicality. Experiment 1 is an extension and refinement of this approach. In this experiment, in addition to requesting ratings of the typicality of faces, we asked our subjects to provide ratings of the attractiveness, likability, familiarity, and memorability of the faces. The attractiveness and likability ratings were taken because the effects of both variables have been suggested to be mediated by typicality (Light et al., 1981; Mueller et al., 1984), and hence these ratings may capture one or more components of it. The judgment of memorability was intended to capture subjects' impressions of the retrievability of the faces, and, thereby, those aspects of typicality thought to be responsible for the effects of distinctiveness.

The judgment of familiarity was intended to capture at least some aspects of the construct of general familiarity and was more problematic to institute. The difficulty with defining this judgment to subjects is that if the definition is too broad, it becomes synonymous with typicality, whereas if it is defined too narrowly, it becomes tantamount to recognition. In the previous study (Vokey \& Read, 1988), we characterized a generally familiar face to our subjects as one that was confusable with the face of someone they knew, a judgment we viewed as being a reasonable com- 
promise between these extremes, but, as it turned out, not without difficulties. First, only a small minority of the faces were judged to be familiar in this sense. Second, it asked subjects to provide this judgment as it related to their personal life histories, which may explain why the reliability of this judgment as assessed across male and female raters was so low relative to other judgments such as that of typicality. To circumvent these problems in the present experiment, subjects were led to believe that some of the faces for which they would be asked to provide familiarity ratings should be familiar to them as those of students from their classes and around the university. Because the faces were not in fact those of students at the university, the subjects were told (correctly) that the photographs they would be asked to make the judgments from were not current, as a way of allaying any concerns that the subjects might have had about the overall low level of familiarity of what were supposed to be familiar faces. These "misrecognitions" would seem to be a relatively direct measure of what is meant by structurally induced familiarity.

\section{Method}

Subjects. Forty-seven university undergraduates served as subject in return for partial course credit in introductory psychology. Twenty-three subjects ( 6 males and 17 females) rated the Grade 10 photographs, and the remaining 24 subjects $(7$ males and $17 \mathrm{fe}-$ males) rated the Grade 12 photographs. The markedly uneven distribution of males and females in these groups reflects that of the larger population of introductory psychology students and is roughly constant across conditions and experiments.

Materials. The stimuli were black and white yearbook photographs of 180 high school students ( 90 males and 90 females) from a distant city. For each individual, both a Grade 10 and a Grade 12 yearbook photograph were obtained and were reproduced as slides. The faces were selected from a larger set of 209 faces of students whose Grade 12 photographs had previously been rated for typicality, general familiarity, and tendency to attract recognition false positives (see Vokey \& Read, 1988). The smaller set was chosen from the extremes of rated typicality within sex of the larger set. Four random orders of the slides within each grade were used.

Procedure. The subjects were tested in groups of 2-5, distributed roughly equally over grade and slide order. The slides were projected at approximately twice life size on the wall in front of the subjects. Each slide was shown for $14 \mathrm{sec}$, with a break of $14 \mathrm{sec}$ every 30 slides. For each slide, the subjects were asked to provide ratings of familiarity, typicality, memorability, likability, and attractiveness. Each rating was made on a 4-point rating scale for which the values 1-4 represented the judgments "sure not ...," "guess not ...," "guess ...," and "sure ...," respectively. To set the cover story for the judgment of familiarity, the subjects were given the following paragraph as the introduction to the task:

You will be presented with a series of photographs of people's faces. These faces are [those] of some of the students who are currently attending the University of Lethbridge in their first year, so we expect that you have seen some, but probably not all of these people in your introductory classes and around the university. However, the photographs are not current; rather they were taken from the students' high school yearbooks-some as far back as when the students were in Grade 10.

A familiar face, then, was defined to the subjects as one that they believed they had seen around the university, but particularly in their first-year classes. As none of the photographs were of people who had ever attended the university, all such judgments were neces sarily false positives. A typical face was further elaborated as one that was "average." A memorable face was defined as one that the subject believed would be relatively easy to remember. For the judgments of likability and attractiveness, the subjects were asked to decide whether the individual depicted was attractive or would be a likable person.

\section{Results}

The mean ratings for each of the judgments as a function of sex of face and grade in which the photograph was taken are shown in Table 1. The data for each rating type were collapsed across subjects and subjected to a two-way analysis of variance with face nested within sex but crossing grade as the random variate. As is shown in Table 1 , the male faces were rated less attractive $[F(1,178)=$ 83.70, $\left.M S_{\mathrm{e}}=0.23\right]$, less likable $\left[F(1,178)=74.52, M S_{\mathrm{e}}\right.$ $=0.11]$, less familiar $\left[F(1,178)=6.08, M S_{\mathrm{e}}=0.06\right]$, less typical $\left[F(1,178)=18.59, M S_{\mathrm{e}}=0.09\right]$, and less memorable $(F<1)$ than the female faces, with the effect reliable at the $\alpha=.05$ level, the significance level used throughout this report, for all but the last judgment. At the same time, Grade 10 faces were rated significantly less likable $\left[F(1,178)=4.05, M S_{e}=0.58\right]$, less familiar $\left[F(1,178)=36.11, M S_{\mathrm{e}}=0.04\right]$, less typical $\left[F(1,178)=9.88, M S_{c}=0.04\right]$, and less memorable $\left[F(1,178)=46.40, M S_{\mathrm{e}}=0.04\right]$, but not less attractive $(F<1)$ than Grade 12 faces. With the exception of the ratings of familiarity, the effects of sex and grade were additive $(F \mathrm{~s}<2.62)$. For familiarity $[F(1,178)=12.33$, $\left.M S_{\mathrm{e}}=0.04\right]$, the interaction was such that the difference between male and female faces was significant for Grade $12\left[F(1,178)=17.71, M S_{\mathrm{e}}=0.05\right]$ but not Grade $10(F<1)$ faces. In general, subjects rated Grade 12 faces more positively than Grade 10 faces, and female faces more positively than male faces, with the two variables being generally additive in effect. As the majority of subjects were female students who had only recently graduated from high school, the results may reflect a bias to rate more positively faces most like those of the majority of raters (cf. Cross, Cross, \& Daly, 1971; McKelvie, 1981).

The data for each grade, again with face as the unit of analysis, were reduced to the correlation matrices shown in Table 2. These correlation matrices were subjected to independent principal components analyses. The first two principal components for each grade were then or-

Table 1

Mean Ratings (on a 4-Point Scale) of Attractiveness, Likability, Familiarity, Typicality, and Memorability of the Faces as a Function of Sex of Face and Grade in Which the Photograph Was Taken

\begin{tabular}{lccccc}
\hline & \multicolumn{2}{c}{ Male } & & \multicolumn{2}{c}{ Female } \\
\cline { 2 - 3 } \cline { 5 - 6 } \multicolumn{1}{c}{ Rating } & Grade 10 & Grade 12 & & Grade 10 & Grade 12 \\
\hline Attractiveness & 2.02 & 2.00 & & 2.43 & 2.51 \\
Likability & 2.40 & 2.48 & & 2.73 & 2.76 \\
Familiarity & 1.64 & 1.69 & & 1.63 & 1.83 \\
Typicality & 2.43 & 2.51 & & 2.57 & 2.63 \\
Memorability & 2.40 & 2.51 & & 2.39 & 2.55 \\
\hline
\end{tabular}


Table 2

Intercorrelation Matrices of the Ratings of the Grade 10 (Lower Triangle) and Grade 12 (Upper Triangle) Faces

\begin{tabular}{|c|c|c|c|c|c|c|c|c|c|}
\hline \multirow[b]{3}{*}{ Rating } & & & & & & \multicolumn{4}{|c|}{ Components } \\
\hline & \multicolumn{5}{|c|}{ Intercorrelations } & \multicolumn{2}{|c|}{1} & \multicolumn{2}{|c|}{2} \\
\hline & 1 & 2 & 3 & 4 & 5 & 10 & 12 & 10 & 12 \\
\hline $\begin{array}{l}1 \text { Attractiveness } \\
2 \text { Likability } \\
3 \text { Familiarity } \\
4 \text { Memorability } \\
5 \text { Typicality }\end{array}$ & $\begin{array}{l}.613 \\
.624 \\
.655 \\
.298 \\
.511\end{array}$ & $\begin{array}{l}.688 \\
.430 \\
.457 \\
.130 \\
.460\end{array}$ & $\begin{array}{l}.381 \\
.296 \\
.180 \\
.260 \\
.415\end{array}$ & $\begin{array}{r}.148 \\
.196 \\
.182 \\
.271 \\
-.423\end{array}$ & $\begin{array}{r}.585 \\
.396 \\
.391 \\
-.372 \\
.376\end{array}$ & $\begin{array}{l}.890 \\
.819 \\
.649 \\
.220 \\
.667\end{array}$ & $\begin{array}{l}.908 \\
.793 \\
.819 \\
.265 \\
.622\end{array}$ & $\begin{array}{r}-.050 \\
.101 \\
.069 \\
.936 \\
-.655\end{array}$ & $\begin{array}{r}.073 \\
-.076 \\
.109 \\
.930 \\
-.712\end{array}$ \\
\hline
\end{tabular}

Note-Between-grade coefficients for each rating are shown along the main diagonal. The components are the first two principal component axes for each grade following a principal components analysis with a varimax rotation.

thogonally rotated to a varimax criterion to produce the "simple structures," also shown in Table 2. Factor scores were computed for each face within each grade to be used in subsequent analyses.

Looking first at the correlations between grades on the ratings (shown on the major diagonal of the correlation matrix in Table 2), it may be seen that most of the correlations are moderate at best, although all are positive and significantly different from zero; only attractiveness managed to achieve a correlation greater than $r=.5$. These moderate correlations are perhaps not surprising, given the major changes in appearance associated with maturation between Grade 10 and Grade 12. Despite the low consistency of the individual ratings across grades, the pattern of correlations among the ratings within grades was quite consistent, as is borne out by the principal components analyses. For the Grade 10 and Grade 12 photographs, the component solutions following varimax rotation are virtually identical. Because the two grades correlated only moderately with one another with respect to the individual ratings, the stability of the principal components solution over grade suggests a stability in the solution beyond that of the particular faces used. This generality of solution is further supported by the finding that the faces' scores on the two components are also not highly correlated over the two grades. The second component correlated only $r(178)=.298$, which while significantly different from zero, does not suggest a great degree of commonality over the two grades. The first component did somewhat better, correlating $r(i 78)=$ .479 over the two grades. Thus, the stability of the structure of the space across grades is more probably attributable to the categorical structure of faces in general than to some transphotograph stability of the faces in the particular face set that we used.

The two components also appear to be easy to interpret. The first component, accounting for $47.5 \%$ and $51.6 \%$ of the variance, respectively, of the Grade 10 and Grade 12 face sets, consists primarily of a positive manifold of the attractiveness, likability, and familiarity ratings and would appear to reflect the construct of general familiarity discussed in the introduction. ${ }^{2}$ The second component, accounting for $26.4 \%$ and $27.9 \%$ of the variance, respectively, of the Grade 10 and Grade 12 face sets, consists of the rating of memorability and correlated not all with the ratings of attractiveness, likability, and familiarity. It would appear to reflect subjects' judgments of explicit retrievability. Typicality, in contrast, loaded roughly equally $(|\bar{r}|=.66)$ but with opposite sign on both components. Given the magnitude of these loadings, typicality would appear therefore to be little more than a mixture of equal parts of both orthogonal components. Regressing typicality on the factor scores for the faces derived from the two components (or, equivalently, summing the squares of the factor loadings) yielded highly significant multiple correlations of $R=.935[F(2,177)$ $\left.=617.13, M S_{\mathrm{e}}=0.01\right]$ and $R=.946[F(2,177)=$ $\left.753.41, M S_{\mathrm{e}}=0.01\right]$, respectively, for the Grade 10 and Grade 12 face sets. That is, roughly $90 \%$ of the variance in the typicality ratings between faces within grades may be accounted for by the summed effects of the two components.

Sex of face was significantly related to the general familiarity component for both the Grade $10[F(1,178)=$ $\left.35.67, M S_{\mathrm{e}}=0.84\right]$ and the Grade $12[F(1,178)=53.79$, $\left.M S_{\mathrm{e}}=0.77\right]$ faces, with male faces receiving the lower component scores on the average. In contrast, sex of face was not significantly related to the memorability component for either grade ( $F \mathrm{~s} \leq 1)$.

\section{Discussion}

The results of the principal components analysis are straightforward. Rated typicality of the faces appears to be composed of roughly equal parts of two orthogonal components, which we will refer to as the components of general familiarity and memorability; increased typicality is associated with increased general familiarity and decreased memorability. Thus, an atypical face is simultaneously both more memorable and less generally familiar than a typical face. The consequences of this result for a model of the effects of typicality on recognition seem equally straightforward. In fact, these results would seem to provide strong support for the two-component model of Light et al. (1979), discussed earlier. Atypical faces are discriminated better on tests of recognition than are typical faces, both because the lower levels of general familiarity result in fewer false-positive responses and because the higher levels of memorability result in enhanced retrieval and, hence, increased hits. We will provide a direct test of this model in subsequent experiments. 
The results also have consequences for explanations of the effects of other variables known to be related to the recognition of faces. Light et al. (1981) reported that rated attractiveness of faces is negatively related to their recognizability and positively related to their typicality (cf. Langlois \& Roggman, 1990). Over a series of different experiments and conditions, attractiveness contributed nothing to the prediction of recognizability beyond that accounted for by typicality (or interitem similarity), leading Light et al. (1981) to argue that the effect of attractiveness on recognizability is mediated by typicality. Light et al. and Mueller et al. (1984) suggested a similar argument for likability, as did Vokey and Read (1988) for the effect of sex of face. Because each of these variables in the present experiment was related only to the first component, the present results suggest that the mediation occurs through the component of general familiarity rather than through the component of memorability or typicality in general.

The finding that attractive or likable faces are less recognizable is counterintuitive. Folk wisdom suggests that highly attractive faces should be easier to remember. Indeed, the common expectation is that the relationship between recognizability and attractiveness should be a $\mathrm{U}$ shaped curvilinear function, with faces of "average" attractiveness being the most difficult to remember. This result, in fact, was reported by Shepherd and Ellis (1973) for recognition following a long (35-day) delay, although shorter delays showed no such effect. Shepherd and Ellis suggested that the recognition advantage that they found for the extremes of attractiveness was a function of distinctiveness; thus, faces of average attractiveness were relatively nondistinct and therefore less memorable than were highly attractive or unattractive faces. Thus, there may be a role for the memorability component of typicality in the mediation of the effect of attractiveness on recognition (Vokey, Read, Moore, \& Sporer, 1991).

Of course, each of these notions is predicated on the assumption that both components, but particularly general familiarity, are responsible for the effect of typicality on recognition. Conceivably, either one component or both components could be unrelated to the recognition of faces. In the next two experiments, we directly investigated the relationship between recognition and the components of general familiarity and memorability.

\section{EXPERIMENT 2 \\ Familiarity, Memorability, and Recognition}

If the effect of typicality on recognition is a function of the two components of general familiarity and memorability, then, in a test of recognition, both components would be expected to be significant contributors to recognition discrimination. According to the Light et al. (1979) model, general familiarity should decrease discrimination while memorability enhances it. Because the components are orthogonal, this may be investigated by regressing recognition discrimination on the factor scores of the faces on the two components to produce a regression equation consisting of their weighted sum. Significance tests of the individual regression coefficients, then, would provide a direct test of the contribution of the corresponding components. ${ }^{3}$ The possibility that the interaction of the two components contributes significantly to recognition discrimination may be investigated by the subsequent hierarchical regression of discrimination on the product vector of the two components. One such interaction would be produced if use of the two components was in a compensatory relation so that, for example, responding on the basis of general familiarity occurred only when memorability was low. Similarly, by hierarchically regressing discrimination on the rated typicality of the faces, one should be able to determine whether there is any residual effect of typicality on recognition discrimination beyond that accounted for by the two componentsthat is, whether general familiarity and memorability provide a complete account of the effect of typicality on recognition.

Recognition discrimination is only one recognition statistic that may be investigated in this way. Overall response bias (i.e., tendency of a face to attract "old" responses) is another. One way to account for the typicality effect in terms of general familiarity is to model it as something that is simply added to the specific familiarity of a face. That is, according to this view, highly typical (i.e., generally familiar) faces would be expected to attract more "old" responses than would atypical faces, both as targets and as distractors. If so, the component of general familiarity should be a significant predictor of response bias. Another possibility, suggested by the model of Light et al. (1979), is that any effect of the component of general familiarity on recognition discrimination might occur as an effect on false alarms without a corresponding effect on hits. Similarly, again as suggested by the model of Light et al. (1979), if the component of memorability corresponds at least in part to the concept of distinctiveness discussed earlier, which in turn facilitates discrimination by increasing the retrievability of targets, we might expect memorability to be associated with hits, but independent of false alarms.

\section{Method}

Subjects. Twenty university undergraduates served as subjects. They participated in return for partial course credit in introductory psychology.

Materials. The materials were slides of 176 of the faces $(88 \mathrm{males}$ and 88 females) used in Experiment 1. Counterbalancing required that the number of faces be a multiple of 16, and 176 was the closest such multiple to the $\mathbf{1 8 0}$ faces used in Experiment 1. As before, a Grade 10 and a Grade 12 slide were available for each face.

Procedure. Subjects were run in groups of 5 people each. Each group was assigned to one of four counterbalancing conditions. These conditions ensured that over subjects each face was used equally often as a target and as a distractor face, and equally often as a Grade 10 and a Grade 12 slide. For initial exposure, the subjects were asked to rate for likability slides of one half of the faces (44 male faces and 44 female faces, one half of each as Grade 10 slides and one half as Grade 12 slides), projected 1 at a time in 
Table 3

\begin{tabular}{|c|c|c|c|c|c|c|c|c|}
\hline \multirow{3}{*}{$\begin{array}{l}\text { Slide } \\
\text { Type }\end{array}$} & \multicolumn{4}{|c|}{ Grade 10} & \multicolumn{4}{|c|}{ Grade 12} \\
\hline & & & & & & & & \\
\hline & Hits & $\begin{array}{l}\text { ralse } \\
\text { Alarms }\end{array}$ & $d_{L}$ & $C_{L}$ & Hits & $\begin{array}{l}\text { False } \\
\text { Alarms }\end{array}$ & $d_{L}$ & $C_{L}$ \\
\hline \multicolumn{9}{|c|}{ Experiment 2: Same Targets } \\
\hline $\begin{array}{l}\text { Male } \\
\text { Female } \\
M\end{array}$ & $\begin{array}{l}.69 \\
.66 \\
.67\end{array}$ & $\begin{array}{l}.30 \\
.25 \\
.27\end{array}$ & $\begin{array}{l}1.71 \\
1.83 \\
1.77\end{array}$ & $\begin{array}{l}0.00 \\
0.20 \\
0.10\end{array}$ & $\begin{array}{l}.65 \\
.65 \\
.65\end{array}$ & $\begin{array}{l}.21 \\
.31 \\
.26\end{array}$ & $\begin{array}{l}1.94 \\
1.51 \\
1.72\end{array}$ & $\begin{array}{l}0.31 \\
0.08 \\
0.19\end{array}$ \\
\hline \multicolumn{9}{|c|}{ Experiment 3: Different Targets } \\
\hline $\begin{array}{l}\text { Male } \\
\text { Female } \\
M\end{array}$ & $\begin{array}{l}.44 \\
.38 \\
.41\end{array}$ & $\begin{array}{l}.30 \\
.30 \\
.30\end{array}$ & $\begin{array}{l}0.57 \\
0.39 \\
0.48\end{array}$ & $\begin{array}{l}0.57 \\
0.68 \\
0.63\end{array}$ & $\begin{array}{l}.36 \\
.39 \\
.38\end{array}$ & $\begin{array}{l}.32 \\
.33 \\
.32\end{array}$ & $\begin{array}{l}0.21 \\
0.22 \\
0.22\end{array}$ & $\begin{array}{l}0.69 \\
0.62 \\
0.66\end{array}$ \\
\hline
\end{tabular}

a random order. Each slide was projected for $5 \mathrm{sec}$, with a break of $5 \mathrm{sec}$ every 11 slides. The subjects then received an unrelated filler task for $30 \mathrm{~min}$ before receiving the recognition task. For the recognition task, the 88 faces previously rated for likability (target faces) were mixed with the remaining 88 faces (distractor faces) and presented at a rate of 1 slide very $5 \mathrm{sec}$, with a break of $5 \mathrm{sec}$ every 11 slides. The subjects responded with a "yes" or "no" to each face to indicate whether they thought it had been one of the faces they had been asked to rate for likability.

\section{Results}

The basic recognition results of the experiment are summarized in the upper panel of Table 3 . For analysis, the data were collapsed across subjects and the resulting hits and false alarms for each face were converted to discrimination $\left(d_{L}\right)$ and bias $\left(C_{L}\right)$ indices based on the logistic distribution (see Snodgrass \& Corwin, 1988). ${ }^{4}$ There was no significant effect of sex of face on either of the latter measures as assessed by one-way analysis of variance within each grade $(F \mathrm{~s}<1)$, so this variable was dropped in subsequent analyses.

Typicality. The standard effect of typicality on recognition was confirmed. Rated typicality was significantly and negatively related to recognition discrimination for both Grade $10\left[r=-.33, F(1,174)=21.21, M S_{\mathrm{e}}=\right.$ 1.76] and Grade $12\left[r=-.40, F(1,174)=33.51, M S_{e}\right.$ $=2.32]$ faces. It was not significantly related to the measure of response bias for Grade 10 faces $[F(1,174)=$ $1.84, M S_{\mathrm{e}}=1.84$ ], but it was marginally related to $C_{L}$ for Grade 12 faces $\left[r=-.14, F(1,174)=3.56, M S_{e}=0.76\right.$, $p=.06]$ in the direction of an increased tendency to attract "old" responses with increased typicality. Typicality also was significantly and positively related to false alarms for both Grade $10\left[r=.28, F(1,174)=15.10, M S_{\mathrm{e}}=\right.$ $0.06]$ and Grade $12\left[r=.37, F(1,174)=26.75, M S_{\mathrm{e}}\right.$ $=0.06]$ faces, and negatively related to hits for Grade 12 $\left[r=-.17, F(1,174)=5.27, M S_{\mathrm{e}}=0.07\right]$ but not Grade $10\left[r=.12, F(1,174)=2.45, M S_{\mathrm{e}}=0.06\right]$ faces.

General familiarity and memorability. The indices of recognition discrimination and bias for the faces were regressed independently within grades on the components of general familiarity and memorability, and the product vector of the two components. In no case did the interaction of the two components contribute significantly to the regression of either $d_{L}$ or $C_{L}(F \mathrm{~s} \leq 1.06)$. That is, in all cases, the effects of the two components may be described as additive (or nonexistent), so the product vector (carrying the interaction) was dropped from the analyses.

The overall regression of the two components for each grade was significant for discrimination $\left[R_{10}=0.31\right.$, $F(2,173)=9.46, M S_{\mathrm{e}}=1.79 ; R_{12}=0.44, F(2,173)=$ $\left.20.48, M S_{\mathrm{e}}=2.25\right]$, but not for bias $(F \mathrm{~s} \leq 1.76)$. For discrimination, for both grades, the effects of both components were also significant. Defining $F$ as a face's score on the general familiarity component, and $M$ as the face's score on the memorability component, the semipartial'correlation coefficients were $r_{d_{L}(F . M)}=-.22[t(173)=$ $3.06]$ and $r_{d_{1}(M . F)}=.22[t(173)=3.10]$, and $r_{d_{L}(F . M)}=$ $-.19[t(173)=2.84]$ and $r_{d_{L}(M . F)}=.39[t(173)=$ 5.79], respectively, for the Grade 10 and Grade 12 faces. The corresponding regression equations for each grade were:

$$
\begin{aligned}
& { }_{10} d_{L}=1.77-.31 F_{10}+.31 M_{10}, \\
& { }_{12} d_{L}=1.73-.32 F_{12}+.66 M_{12} .
\end{aligned}
$$

As with the regression analyses of discrimination and bias, the multiple regression analyses of hits and false alarms yielded no significant interaction of the two components for either grade $(F \mathrm{~s}<1)$, so again the product vector was dropped from the regressions. As with the simple regressions of hits and false alarms on typicality, the overall regression of the two components for each grade was significant for false alarms $\left[R_{10}=0.26, F(2,173)=\right.$ $6.08, M S_{\mathrm{c}}=0.06 ; R_{12}=0.36, F(2,173)=12.55, M S_{\mathrm{e}}$ $=0.06]$, but was significant for hits only for Grade 12 faces $\left[R_{10}=0.13, F(2,173)=1.42, M S_{e}=0.07, p=\right.$ $\left..25 ; R_{12}=0.25, F(2,173)=5.60, M S_{e}=0.07\right]$. For false alarms, the effects of both components were significant for both grades, but only the memorability component was significant for hits on Grade 12 faces. Defining $f$ as the proportion of false alarms that a face received, 
and $h$ as the proportion of hits that a face received, the semipartial correlations of the components with Grade 10 false alarms were $r_{f(F . M)}=.20[t(173)=2.74]$ and $r_{f(M . F)}=-.16[t(173)=2.17]$. For Grade 12 faces, the semipartial correlations of the components with the false alarms were $r_{f(F . M)}=.23[t(173)=3.22]$ and $r_{f(M . F)}=$ $-.28[t(173)=3.90]$; the semipartial correlation for the Grade 12 hits with the memorability component was $r_{h(M . F)}=-.24[t(173)=3.31]$. The corresponding regression equations were: ${ }^{5}$

$$
\begin{aligned}
& f_{10}=.28+.05 F_{10}-.04 M_{10}, \\
& f_{12}=.26+.06 F_{12}-.07 M_{12}, \\
& h_{12}=.65+.07 M_{12} .
\end{aligned}
$$

One last result should be noted. Each of the regression analyses within grades was performed again, but with typicality included as a third predictor. The increment in variance accounted for was then tested for significance. In no case did the inclusion of typicality significantly improve prediction beyond that accounted for by the two component variables. The maximum $F$ for the recognition of Grade 10 faces was found for the improvement in the prediction of false alarms $\left[F(1,172)=2.94, M S_{e}=0.06\right.$, $p=.09]$. For Grade 12 faces, the maximum $F$ was found for the prediction of $C_{L}\left[F(1,172)=2.28, M S_{\mathrm{e}}=0.76\right.$, $p=.13]$.

\section{Discussion}

The effect of typicality on recognition in Experiment 2 confirmed that found in other studies. The general effect is that increasing typicality reduces recognition discrimination by increasing false alarms and, at least for the Grade 12 faces in this experiment, by reducing hits. There was also a suggestion for the recognition of the Grade 12 faces that increasing typicality was associated with increasing familiarity of the faces generally, independently of their status as targets or distractors.

The two components isolated in Experiment 1 did an excellent job of capturing this pattern of relations for typicality. As shown in Regression Equations 1.1 and 1.2, the two components affected recognition discrimination in opposite directions. For the recognition of the Grade 10 faces, for example, for every unit increase in general familiarity, $d_{L}$ was reduced by an average of 0.31 units (i.e., roughly one third of a standard deviation); but for every unit increase in memorability, $d_{L}$ was increased by same the average amount. The effects for the recognition of the Grade 12 faces were similar, except that the rate of increase associated with a unit change in memorability was twice that of the decrease associated with general familiarity. Thus, we would appear to have strong evidence that the effect of typicality on recognition discrimination is mediated by the two components of general familiarity and memorability. The fact that the effects of the two components were apparently additive and the fact that typicality contributed nothing further to these regressions beyond that already accounted for by the two components suggest that the effect of typicality on recognition discrimination may be nothing more than the simple summed effect of the two components.

The analyses of hits and false alarms as a function of the two components help isolate the locus of effect of the two components. One part of the effect of the memorability component would appear to occur through enhanced retrieval of highly memorable targets, as suggested by the model of Light et al. (1979) and by most explanations of typicality effects in terms of distinctiveness. As shown in Regression Equation 3.1, for Grade 12 faces, for every unit increase in memorability, the proportion of hits increased by an average of .07 . That this effect of the memorability component on hits was found only for Grade 12 faces echoes the result that an effect of typicality on hits also was found only for these faces, and supports the idea that, at least for some face sets, encoded distinctiveness and consequent enhanced retrieval is one aspect of the typicality effect. As to why the effect was limited to Grade 12 faces, we can only speculate, but it may be related to the fact that the Grade 12 faces as a set were more familiar to the subjects (i.e., in that they were more similar in age to the subjects) than the Grade 10 faces. Some studies have yielded an own-race advantage in recognition (see Shepherd, 1981), and McKelvie (1981) has reported a same-sex advantage for females that appears to be further dependent on the similarity of the faces (in age and nationality) to the face of the recognizer. The current result may be another example of the same phenomenon. If so, it suggests a mechanism for the effect in terms of encoded distinctiveness. Presumably, the greater overall familiarity of the stimulus set allows the recognizer to take advantage at encoding and retrieval of the variations in distinctiveness within the set that otherwise fail to be noticed or utilized. The greater overall familiarity of these items, however, should also incur the cost of greater false alarms, and the precise balance of these two factors may be responsible for the phenomenon's being somewhat elusive.

No effect of the general familiarity component was found for hits. The familiarity component, however, was important for false alarms for both grades of faces. As is shown in the Regression Equations 2.1 and 2.2, for every unit increase in general familiarity, false alarms increased by an average of $5 \%$ or $6 \%$.

The memorability component was also important for false alarms to faces from both grades. As shown in Regression Equations 2.1 and 2.2, increases in memorability were associated with reductions in false alarms; for every unit increase in memorability, the proportion of false alarms decreased by an average of .04 for Grade 10 faces and by an average of .07 for Grade 12 faces. Indeed, as the effect of memorability on hits was limited to one grade, this effect on false alarms may be considered to be the main effect of the memorability component.

An effect of this type for memorability or distinctiveness has not often been postulated in explanations of the typicality effect or in discussions of recognition in gen- 
eral, which have favored instead effects on retrieval. Brown, Lewis, and Monk (1977), however, advanced just such an argument for the effect of word frequency on recognition. They discuss it in terms of what they refer to as "negative recognition"- how we know that we have not experienced something previously. They reject as inadequate the simple absence of positive memory (i.e., retrieval) as an explanation for negative recognition, and they argue that in situations of low or nonexistent retrieval (such as with distractors on tests of item recognition), subjects assess the memorability (i.e., retrievability potential) of the test item. If the item is assessed to be high in memorability, rejection of the item is quite likely, because the lack of retrieval for what should be an easily retrieved item is strong evidence that the item should not be accepted as a target; it would take, say, exceptionally high levels of familiarity to the same item to overcome this evidence from memorability. For items low in memorability, the lack of retrieval is at best weak evidence that the item is not a target, and subjects presumably use other sources of information (e.g., feelings of familiarity) to make their decisions. Thus, distractors of high memorability (e.g., low-frequency words, or atypical faces), all other things being equal, should receive fewer false alarms.

The present results for the memorability component provide strong support for the Brown et al. (1977) memorability hypothesis. Indeed, in the absence of some explanation such as this, it is not apparent why there should be an effect of the memorability component on distractors. The combined results on false alarms of the general familiarity and memorability components suggest further that the two processes function in opposition in the control of false alarms and, ultimately, discrimination. Thus, the tendency for items high in structurally induced familiarity to attract false alarms may be ameliorated by high memorability. By the same token, items low in memorability may be responded to almost entirely in terms of their levels of familiarity. Note that we are not suggesting that the use of familiarity information is contingent on memorability (or the opposite). Rather, as is suggested by the consistent failure to find an interaction of these two components, the subjects' recognition decisions (for distractors) appear to reflect the simple sum of these two oppositely weighted sources of information. The typicality effect on recognition, then, in large part, appears to be a consequence of the fact that for faces generally, these two sources of information are usually correlated with typicality.

It is important to note that the two components do not provide an account of recognition discrimination per se. Indeed, the bulk of the recognition discrimination in this experiment, as well as the bulk of hits and false alarms, are to be found in the intercepts of the regression equations, rather than the regression coefficients associated with the components. But the same is true of the effect of typicality. It too was only moderately related to recognition-but to the extent that it was, our two components accounted completely for it.

\section{EXPERIMENT 3 Changed Targets}

The next experiment was intended to test the generality of the two-component account of the effect of typicality on recognition. Many face recognition experiments, including Experiment 2, are carried out with what may be referred to as the standard paradigm: Subjects are exposed to a set of photographs of faces and then at test are asked to discriminate the same photographs of the faces from photographs of faces not shown previously (see Shapiro \& Penrod, 1986). A major criticism of the use of this paradigm for the investigation of face recognition is that it confounds recognition of a face with the recognition of one photograph or example of the face (see, e.g., Bartlett \& Leslie, 1986; Bruce, 1982; Hay \& Young, 1982; Patterson, 1978). It is not at all clear that the processes that underlie the recognition of specific photographs of faces are necessarily the same or even react the same to various manipulations as do the processes that support the recognition of faces more generally. Simple changes in pose or expression over study and test can have substantial effects on face recognition (see, e.g., Bartlett \& Leslie, 1982; Bruce, 1982; Krouse, 1981; Woodhead, Baddeley, \& Simmonds, 1979), as can the changes associated with aging (Seamon, 1982) or explicit disguises (Patterson \& Baddeley, 1977). Of more direct concern for our current purposes, however, is the possibility that, such transformations may interact with typicality or the component variables identified in the previous experiment. Read, Vokey, and Hammersley (1990), for example, found that although the variable of exposure duration demonstrated the expected positive relationship with recognition accuracy for highly similar study-test photograph pairs of the same face (analogous to the standard paradigm), low-similarity pairs actually demonstrated the reverse relationship. Few studies have been done of the effect of typicality on recognition with any procedure other than the standard paradigm. Experiment 3 was an attempt to fill that gap, and to investigate the applicability to this situation of the component analysis exploited in Experiment 2.

\section{Method}

Subjects. Twenty people from the population used in Experiment 2 served as subjects.

Materials and Procedure. The materials were the same as those used in Experiment 2. The only change in procedure was that the slide for each target face at test was a different photograph of the same individual taken from the alternate grade category. Thus, a face that appeared as a Grade 10 (or Grade 12) slide during initial exposure was presented as the corresponding Grade 12 (or Grade 10) slide of the face at test.

\section{Results}

The basic results of the recognition test, analyzed as in the previous experiment, are shown in the lower panel of Table 3. The unit of analysis was the test photograph; that is, we were concerned with how the ratings or component scores of the test photograph (which is of a face from one grade) affected the recognition of the depicted 
face (which was presented as a photograph from the alternate grade at study). Relative to the recognition performance in Experiment 2, the use of different test photographs in the present experiment had two immediate and unsurprising effects: subjects found fewer of the test slides overall to be familiar, as given by the significantly greater mean value of $C_{L}\left[F(1,174)=86.67, M S_{\mathrm{e}}=0.50\right]$, and recognition discrimination was clearly inferior, as indexed by the markedly lower mean $d_{L}\left[F(1,174)=169.15, M S_{\mathrm{e}}\right.$ $=2.04]$. In fact, although the mean $d_{L}$ for the Grade 10 test faces was significantly greater than zero $[t(175)=$ 4.67], the recognition performance on the Grade 12 test faces was only marginally significantly better than no discrimination at all $[t(175)=2.01, p=.05]$.

Typicality. Despite the use of different test photographs and the low overall level of discrimination, the standard effect of typicality on recognition was found for some of the measures. Recognition discrimination $\left(d_{L}\right)$ for Grade 12 test faces was significantly and negatively related to typicality $\left[r=-.25, F(1,174)=11.65, M S_{\mathrm{e}}=1.90\right]$. Grade 10 test faces, however, showed no such effect $(r=$ $-.04, F<1$ ). As in the previous experiment, typicality was positively related to false alarms for both Grade 10 $\left[r=.37, F(1,174)=27.74, M S_{c}=0.05\right]$ and Grade 12 $\left[r=.45, F(1,174)=43.54, M S_{e}=0.06\right]$ test faces. However, it also was positively related to hits for both Grade $10\left[r=.31, F(1,174)=18.10, M S_{\mathrm{e}}=0.07\right]$ and Grade $12\left[r=.16, F(1,174)=4.84, M S_{e}=0.08\right]$ test faces. That is, increased typicality of test faces was generally associated with an increased tendency to attract positive ("old") responses, regardless of the faces' status as targets or distractors. This tendency was also reflected in the significant positive relationship between typicality and response bias $\left(C_{L}\right)$ for both Grade $10[r=.41$, $\left.F(1,174)=34.91, M S_{\mathrm{e}}=0.73\right]$ and Grade $12[r=.38$, $\left.F(1,174)=29.4, M S_{\mathrm{e}}=0.81\right]$ test faces.

General familiarity and memorability. The multiple regression analyses involving the two component variables generally tracked the pattern of results for typicality. As was true for the results of Experiment 2, in none of the analyses did the interaction of the components contribute significantly to the regression on $d_{L}, C_{L}$, hits, or false alarms $(F \mathbf{s}<1)$, and the product vector was therefore dropped from the analyses. Furthermore, as was found in Experiment 2, typicality failed to contribute significantly to the regression for any of the recognition measures beyond that accounted for by the two component variables [for Grade 10 test faces, the maximum $F(1,172)$ $=1.01, M S_{\mathrm{e}}=1.86, p=.62$, was found for discrimination; for Grade 12 test faces, the maximum $F(1,172)=$ 2.91, $M S_{\mathrm{e}}=0.06, p=.09$, was found for false alarms].

As with the regression on typicality, the regression of $d_{L}$ on the two component variables was significant only for the Grade 12 test faces $\left[R_{12}=0.25, F(1,173)=5.86\right.$, $\left.M S_{\mathrm{e}}=1.91 ; R_{10}=0.07, F<1\right]$, to which both the general familiarity $\left[r_{d_{L}(F . M)}=-.18, t(173)=2.50\right]$ and the memorability $\left[r_{d_{L}(M . F)}=.18, t(173)=2.39\right]$ compo- nents contributed significantly. The regression equation for the Grade 12 test faces was: 6

$$
{ }_{12} d_{L}=0.22-.26 F_{12}+.25 M_{12} .
$$

Again, as with the regression on typicality, both Grade 12 $\left[R_{12}=0.37, F(1,173)=11.01, M S_{\mathrm{e}}=0.82\right]$ and Grade $10\left[R_{10}=0.44, F(1,173)=14.62, M S_{\mathrm{e}}=0.71\right]$ test faces showed a significant regression of $C_{L}$ on the two component variables, and for both grades, both components contributed significantly to the regression [for Grade 10 test faces, $r_{C_{L}(F . M)}=-.34, t(173)=4.97$, and $r_{C_{L}(M . F)}=.28, t(173)=4.06$; for Grade 12 test faces, $r_{C_{L}(F . M)}=-.27, t(173)=3.84$, and $r_{C_{L}(M . F)}=$ $.25, t(173)=3.54]$. The regression equations were:

$$
\begin{aligned}
& { }_{10} C_{L}=0.62-.32 F_{10}+.26 M_{10}, \\
& { }_{12} C_{L}=0.66-.26 F_{12}+.24 M_{12} .
\end{aligned}
$$

The multiple regression of hits on the two component variables was not significant for Grade 12 test faces $\left[R_{12}\right.$ $\left.=0.15, F(1,173)=2.039, M S_{\mathrm{e}}=0.08, p=.13\right]$ but was significant for Grade 10 test faces $\left[R_{10}=0.31\right.$, $\left.F(1,173)=9.01, M S_{e}=0.07\right]$. Both component variables of the latter regression analysis contributed significantly to the regression, but in a peculiar way. General familiarity was positively associated with hits $\left[r_{h(F . M)}=\right.$ $.23, t(173)=3.23$ ] , and memorability was negatively associated with hits $\left[r_{h(M . F)}=-.20, t(173)=2.77\right]$-the pattern anticipated for false alarms. The regression equation was: ${ }^{7}$

$$
h_{10}=.41+.07 F_{10}-.06 M_{10} .
$$

For false alarms, the multiple regression on the component variables was significant for both grades of test faces $\left[R_{10}=0.41, F(1,173)=17.22, M S_{\mathrm{e}}=0.06 ; R_{12}\right.$ $\left.=0.44, F(1,173)=20.55, M S_{\mathrm{c}}=0.06\right]$, and for both grades, both components contributed significantly with the expected pattern to the regression [for Grade 10 test faces, $r_{f(F . M)}=.31, t(173)=4.48$, and $r_{f(M . F)}=-.26$, $t(173)=3.81$; for Grade 12 test faces, $r_{f(F . M)}=.34$, $t(173)=4.92$, and $\left.r_{f(M . F)}=-.29, t(173)=4.21\right]$. The regression equations were:

$$
\begin{aligned}
& f_{10}=.30+.08 F_{10}-.07 M_{10} \\
& f_{12}=.33+.09 F_{12}-.08 M_{12} .
\end{aligned}
$$

\section{Discussion}

Despite the differences in recognition and the effect of typicality on recognition between Experiments 2 and 3, the two component variables of general familiarity and memorability in this experiment again tracked the typicality effects. For example, although recognition was best for Grade 10 test faces, the effect of typicality on recognition occurred primarily with Grade 12 faces, which were also the faces to which effects of the two components on discrimination were limited. As before, however, the general familiarity component was associated with 
reduced discrimination and the memorability component with enhanced discrimination (see Equation 4.1), confirming that the effects hold even with changed photographs. Thus, our two-component account of the effect of typicality on recognition would appear to have considerable generality, consistently tracking the effects of typicality despite marked changes in recognition and the effects of typicality both across experiments and between sets of photographs within experiments.

Unlike in Experiment 2, typicality and both components were also related to overall response bias, reflecting the fact that much of the typicality-related variation in responding to the faces in Experiment 3 was unrelated to discrimination. As shown in Equations 5.1 and 5.2, increases in familiarity were associated with a lowering of the response criterion (i.e., an increased likelihood of attracting an "old" response), while increases in memorability resulted in an increased response criterion and, therefore, in a reduced likelihood of attracting an "old" response. In fact, this pattern appears to capture the whole of the effect of typicality and the two components on responses to the Grade 10 test faces; insofar as these effects were concerned, targets were responded to no differently than distractors (see Equation 6.1). We can only speculate as to the source of this result, but it may be related to the finding in Experiment 2 that a positive effect of memorability on hits was found only for Grade 12 faces. It will be remembered that the Grade 10 test faces in Experiment 3 were used to test subjects' memory for Grade 12 training faces. We suggested that the results for these photographs in Experiment 2 arose because the greater overall familiarity of these Grade 12 faces resulted in the subjects' being better able to make use of the distinctive (i.e., memorable) characteristics of the faces. If, however, these distinctive characteristics are for the most part unique to faces from that grade, the subjects' emphasis on them at study would be of little use to them as distinctive retrieval cues at test with the Grade 10 photographs. Worse yet, according to Brown et al.'s (1977) memorability hypothesis, the different (but less effective) set of memorable characteristics of the Grade 10 test faces could lead to the rejection of the targets as well as the distractors (see Read et al., 1990, for a related account of the effect of exposure duration and face similarity).

As in Experiment 2, the two components were significantly related to the production of false alarms for both grades of test faces, accounting with no significant residual for the effect of typicality on false alarms. As shown in Regression Equations 7.1 and 7.2, unit increases in general familiarity increased false alarms by $8 \%$ or $9 \%$, but unit increases in memorability were associated with decreases in false alarms of roughly the same amount. Again, this negative effect of the memorability component on false alarms appears to be the main effect of memorability, and it provides strong support for the Brown et al. (1977) memorability hypothesis. In fact, contrary to most explanations of the typicality effect that emphasize the benefits of distinctiveness on retrieval, there was no positive effect of memorability on hits in this experiment. We suspect that, at least to some extent, this lack of a distinctiveness effect on hits reflects the fact that the component scores of the test faces rather than the study faces are used in the analyses. Nevertheless, the general results suggest that the emphasis on distinctiveness as of primary benefit to retrieval may have been overdone.

\section{GENERAL DISCUSSION}

The results of the present three experiments support three major conclusions. First, in Experiment 1 it was demonstrated that typicality can be decomposed into two independent components, which we have characterized as general familiarity and memorability. Both of these components are thought to reflect the categorical structure of the similarity space of faces. Thus, an atypical face in both low in structurally induced familiarity (because it resembles few other faces in the recognizer's experience) and high in memorability. Subsidiary to this conclusion is the possibility that the effects of other facial attributes, such as attractiveness and likability, that have been suggested to be mediated by typicality may in fact be mediated by only one component of it. Second, the results of both Experiments 2 and 3 demonstrated that the effects of typicality on recognition-although themselves variable over same as opposed to different photographscan be accounted for simply by the additive effects of the two components with no significant residual; general familiarity of the test faces decreases recognition discrimination, whereas the memorability of the test faces enhances it. Third, although there was a positive effect of the memorability component on hits and, hence, retrieval for the Grade 12 faces in Experiment 2, the major effect of this component for both grades in both Experiments 2 and 3 occurred in the reduction of false alarms. This result argues strongly for the need for notions such as the Brown et al. (1977) memorability hypothesis in any account of face recognition or recognition generally.

The model of recognition that we favor is similar to many two-process models that have been proposed (see, e.g., Mandler, 1980). The basic idea is that the familiarity arising from some specific prior exposure is not intrinsically separable from the structurally induced familiarity that arises from a lifetime of experience with similar exemplars. The fundamental difficulty facing the recognizer, then, is how to discriminate specifically familiar events from those that are merely generally familiar. If specific familiarity when added to existing levels of familiarity can be assumed on the average to exceed some background level of general familiarity, then one approach, as suggested by most strength models of recognition, is to set some appropriate criterion of familiarity for responding and to accept the inevitable errors of omission and commission. Another approach is to ignore familiarity altogether, and to rely on the retrieval of the context and elaboration of the original encounter. Subjects apparently rarely resort exclusively to one or another of 
these approaches, favoring instead the additive mixture of both sources of information (see, e.g., Mandler, 1980). The current results suggest further, however, that subjects include the assessment of retrievability coupled with the absence of retrieval as a negatively signed component in this sum.

The typicality effect with the recognition of faces is just one example of a larger set of common recognition effects with other materials and stimulus variables that Glanzer and Adams $(1985,1990)$ have referred to collectively as the mirror effect. This effect refers to the regularity that, for many domains, if one class of items (e.g., atypical faces or low-frequency words) is recognized better than some other class (e.g., typical faces or high-frequency words), the superior class is recognized better as being old when it is old and as being new when it is new. The mirror effect appears to hold over different recognition paradigms (e.g., yes/no, forced choice, etc.) and over many different stimulus variables, including concreteness and meaningfulness, in addition to those already mentioned (Glanzer \& Adams, 1985). Recently, Glanzer and Adams (1990) have demonstrated that, in addition to stimulus variables, the mirror effect generalizes to operations that subjects perform on the memory materials. For example, the requirement that subjects first reverse some words spelled backward before pronouncing them results in the mirror effect on recognition for reversed as opposed to nonreversed words; reversed words result in both fewer false alarms and greater hits than do nonreversed words. Glanzer and Adams (1990) noted of this last result that it suggests that "any variable that affects recognition, not just stimulus variables, will produce the effect"' (p. 12).

Glanzer and Adams (1990) discuss the mirror effect in terms of what they call "attention/likelihood theory." Although the details of this theory are beyond the scope of the present paper, the thrust of the theory, as in Brown et al.'s (1977) memorability hypothesis, is that subjects compare their current information or knowledge about a test item to their expectations about or models of target and distractor items, and base their responses on the results of this comparison. The results of the current series of experiments on the typicality effect and the recognition of faces provide strong support for theories of this type.

\section{REFERENCES}

Bartlett, J. C., Hurry, S., \& Thorley, W. (1984). Typicality and familiarity of faces. Memory \& Cognition, 12, 219-228.

Bartlett, J. C.. Lesue, J. E. (1986). Aging and memory for faces versus single views of faces. Memory \& Cognition, 14, 371-381.

Brown, J., Lew1s, V. J., \& Monk, A. F. (1977). Memorability, word frequency and negative recognition. Quanterly Joumal of Experimental Psychology, 29, 461-473.

BruCE, V. (1982). Changing faces: Visual and non-visual processes in face recognition. British Journal of Psychology, 73, $105-116$.

Cohen, M. E., Carr, W. J. (1975). Facial recognition and the von Restorff effect. Bulletin of the Psychonomic Society, 6, 383-384.

Courtois, M. R., MUrller, J. H. (1981). Target and distractor typicality in facial recognition. Journal of Applied Psychology, 66, 639-645.
Cross, J. F., Cross, J., \& Daly, J. (1971). Sex, race, age, and beauty as factors in recognition of faces. Perception \& Psychophysics, 10, 393-396

Ellis, H. D., Shepherd, J. W., Gibung, F., \& Shepherd, J. (1988). Stimulus factors in face learning. In M. M. Gruneberg, P. E. Morris. \& R. N. Sykes (Eds.), Practical aspects of memory: Current research and issues: Vol. I. Memory in everyday life (pp. 136-143). Chichester, U.K.: Wiley.

Glanzer, M., Adams, J. K. (1985). The mirtor effect in recognition memory. Memory \& Cognition, 13, 8-20.

Glanzer, M., Adams, J. K. (1990). The mirtor effect in recognition memory: Data and theory. Journal of Experimental Psychology: Leaming, Memory, \& Cognition, 16, 5-16.

GoING, M., \& READ, J. D. (1974). The effect of uniqueness, sex of subject and sex of photograph on facial recognition. Perceptual \& Motor Skills, 39, 109-110.

HAY, D. C., Young, A. W. (1982). The human face. In A. W. Ellis (Ed.), Normality and pathology in cognitive functions (pp. 173-202). London: Academic Press.

Krouse, F. L. (1981). Effects of pose, pose change, and delay on face recognition performance. Joumal of Applied Psychology, 66, 651-654.

LANGLots, J. H., RoGgman, L. A. (1990). Attractive faces are only average. Psychological Science, 1, 115-121

Light, L. L., Hollander, S., Kayra-Stuart, F. (1981). Why attractive people are harder to remember. Personality \& Social Psychology Bullerin, 7, 269-276.

light, L. L., Kayra-Stuart, F., Hollander, S. (1979). Recognition memory for typical and unusual faces. Joumal of Experimental Psychology: Human Learning \& Memory, 5, 212-228.

MANDLER, G. (1980). Recognizing: The judgment of previous occurrence. Psychological Review, 87, 252-271.

MCKELVIE, S. J. (1981). Sex differences in memory for faces. Jourmal of Psychology, 107, 109-125.

Mueller, J. H., HeEsacker, M., \& Ross, M. J. (1984). Likability of targets and distractors in facial recognition. American Joumal of Psychology, 97, 235-247.

Patterson, K. E. (1978). Person recognition: More than a pretty face. In M. M. Gruneberg, P. E. Morris, \& R. N. Sykes (Eds.), Practical aspects of memory (pp. 228-235). New York: Academic Press.

Patterson, K. E., Baddeley, A. D. (1977). When face recognition fails. Joumal of Experimental Psychology: Human Leaming \& Memory, 3, 406-417.

PEdhazUR, E. J. (1982). Multiple regression in behavioral research (2nd ed.). New York: Holt, Rinehart \& Winston.

ReAd, J. D., Vokey, J. R., Hammersley, R. (1990). Changing photos of faces: Effects of exposure duration and photo similarity on recognition and the accuracy-confidence relationship. Joumal of Experimental Psychology: Leaming, Memory, \& Cognition, 16, 870-882.

SenMon, J. G. (1982). Dynamic facial recognition: Examination of a natural phenomenon. American Joumal of Psychology, 95, 363-381

Shaptro, P. N., Penrod, S. (1986). Meta-analysis of facial identification studies. Psychological Bulletin, 100, 139-156.

SHEPHERD, J. W. (1981). Social factors in face recognition. In G. Davies, H. Ellis, \& J. Shepherd (Eds.), Perceiving and remembering faces (pp. 57-79). London: Academic Press.

SHEPHERD, J. W., \& ElLIS, H. D. (1973). The effect of attractiveness on recognition memory for faces. American Journal of Psychology, 86, 627-633.

Snodgrass, J. G., Corwin, J. (1988). Pragmatics of measuring recognition memory: Applications to dementia and amnesia. Joumal of Experimental Psychology: General, 117, 34-50.

VAlENTINE, T., BruCE, V. (1986a). The effects of distinctiveness in recognising and classifying faces. Perception, 15, 525-535.

VAlentine, T., Bruce, V. (1986b). Recognizing familiar faces: The role of distinctiveness and familiarity. Canadian Journal of Psychology, 40, 300-305.

VoKeY, J. R., READ, J. D. (1988). Typicality, familiarity and the recognition of male and female faces. Canadian Joumal of Psychology, 42, 489-495.

VoKeY, J. R., ReAD, J. D., Moore, L., \& SPORER, S. L. (1991). Attractiveness and typicality in the recognition of faces. Paper presented 
at the annual meeting of the Canadian Psychological Association, Calgary.

WINOGRAD, E. (1981). Elaboration and distinctiveness in memory for faces. Joumal of Experimental Psychology: Human Leaming \& Memory, 7, 181-190.

WoOdheAd, M. M., BAdDeley, A. D., Simmonds, D. C. (1979).

On training people to recognize faces. Ergonomics, 22, 333-343.

\section{NOTES}

1. As a theoretical construct, the concept of structural typicality is clear enough, but its instantiation or operational definition for faces varies widely across studies. In many face recognition studies, typicality has been assessed by asking subjects to indicate which of the faces they believe are unusual or distinctive, so that being typical would be the semantic anchor or default judgment (e.g., Going \& Read, 1974; Vokey \& Read, 1988). Others have used such diverse measures as the objective memorability of the faces (Ellis, Shepherd, Gibling, \& Shepherd, 1988) or the subjects' assessment of the rank order of the ease of spotting each face in a crowd (Valentine \& Bruce, 1986a). These different measures are not necessarily equivalently representative of the underlying construct of structural typicality and may correlate poorly with one another. Furthermore, some of them (e.g., distinctiveness or familiarity) presuppose one or another putative source of the effect of typicality on recognition, whereas others, such as differences in objective memorability, are in fact the data to be explained. To be fair, many of these measures were not selected with the construct of structural typicality in mind. However, despite the disparity, each of the different measures probably captures one or more aspects of structural typicality. The identification of these differing aspects and their effect on recognition is the focus of the present investigation.

2. Because ratings of attractiveness correlated more highly with the component we have labeled as "general familiarity" than did either the likability ratings or the familiarity judgments (or the typicality ratings, for that matter), it might reasonably be asked why we did not simply call the component "attractiveness." The overriding reason is that the construct of general familiarity is consistent with both the data and with broader theory; as outlined in the introduction to the paper, general familiarity is a common component of explanations of the typicality effect, and it is logically derived from considerations of categorical structure. In contrast, we are unaware of any explanation of the typicality effect or face recognition in general that posits attractiveness as a fundamental theoretical construct. Furthermore, although it has been proposed that general familiarity mediates attractiveness (e.g., Light et al., 1979), to our knowledge, no one has found it necessary to suggest the converse. But we have more specific reasons to prefer the label of "general familiarity" over "attractiveness." If the component were just attractiveness, it would be an attractiveness different from the subjects' direct ratings of it; as shown in Table 2, with the lone exception of the Grade 10 familiarity ratings, higher correlations for both grades with likability and familiarity (and typicality) were found for the component than were found with the ratings of attractiveness directly. Thus, the component appears to be something that all three ratings (or four, if typicality is included) share to a large degree, but of which none is a direct measure. General familiarity is certainly a reasonable interpreta- tion of the common component underlying this positive manifold of correlational clustering of the ratings, and the component also behaves as one would expect of general familiarity in the subsequent tests of recognition. It is not at all clear what one should expect of "general attractiveness" as a component in face recognition.

3. The vicissitudes of rounding error and numeric precision in the generation of factor scores for the faces from the factor score weights and scores on each of the variables resulted in the vectors of the factor scores within each grade not being perfectly orthogonal. Reducing the number of faces used from the original 180 to the 176 in this experiment exacerbated the problem further, although at its highest it achieved a correlation of only $r=.02$ for the two component vectors for the Grade 12 faces. Still, in acknowledgment of the slight confounding, the regression coefficients resulting from standard multiple regression analyses that we report are semipartial regression coefficients and therefore are slightly different from the simple regression coefficients. Other effects (e.g., the interaction of the two components) also were investigated by using hierarchical regression and were tested for their increment in $R^{\mathbf{2}}$ beyond that given by the regression of the two components (e.g., Pedhazur, 1982).

4. Following Snodgrass and Corwin (1988), these measures were computed as follows for each face:

$$
\begin{gathered}
h=\frac{\text { Hits }+.5}{\text { Targets }+1}, \quad f=\frac{\text { False alarms }+.5}{\text { Distractors }+1}, \\
d_{L}=\ln \left(\frac{h(1-f)}{(1-h) f}\right), \quad C_{L}=\frac{1}{2}\left[\ln \left(\frac{(1-f)(1-h)}{h f^{\prime}}\right)\right] .
\end{gathered}
$$

$C_{L}$ represents the signed distance of the decision criterion from the midpoint between the means of the target and distractor distributions; a $C_{L}$ of zero indicates no bias, and positive values index a bias against calling the faces "old."

5. Even though the overall regression was not significant for the Grade 10 hits, the pattem of weights for this and other nonsignificant regressions may be of interest. Accordingly, the regression equation for Grade 10 hits was:

$$
h_{10}=.67-.02 F_{10}+.03 M_{10} .
$$

Similarly, the regression equation for Grade 12 hits, including the nonsignificant coefficient for the familiarity component, was:

$$
h_{12}=.65-.01 F_{12}+.07 M_{12} \text {. }
$$

6. The nonsignificant regression equation for Grade 10 discrimination was:

$$
{ }_{10} d_{L}=.48-.06 F_{10}+.07 M_{10} .
$$

7. The nonsignificant regression equation for Grade 12 hits was:

$$
h_{12}=.38+.03 F_{12}-.03 M_{12} \text {. }
$$

(Manuscript received April 22, 1991; revision accepted for publication October 2, 1991.) 